\title{
Relações público-privadas no sistema de saúde do Chile: regulação, financiamento e provisão de serviços
}

\author{
Public-private relations in Chile's health system: regulation, \\ funding and service delivery
}

Suelen Carlos de Oliveira (https://orcid.org/0000-0002-0090-2341) ${ }^{1}$ Cristiani Vieira Machado (https://orcid.org/0000-0002-9577-0301) ${ }^{1}$ Alex René Alarcón Hein (https://orcid.org/0000-0001-7163-9280) ${ }^{2}$ Patty Fidelis de Almeida (https://orcid.org/0000-0003-1676-3574) ${ }^{3}$

${ }^{1}$ Escola Nacional de Saúde Pública Sergio Arouca, Fundação Oswaldo Cruz. R. Leopoldo Bulhões 1.480, Manguinhos. 21041-210 Rio de Janeiro RJ Brasil. suelen.c.oliveira@gmail.com ${ }^{2}$ Escuela Nacional de Salud Pública Salvador Allende, Universidad de Chile.

Santiago Chile.

${ }^{3}$ Instituto de saúde

Coletiva, Departamento de

Planejamento em Saúde,

Universidade Federal

Fluminense. Niterói RJ Rio

de Janeiro.

\begin{abstract}
This article analyzes the configuration of public-private relations in Chile's health system between 2000 and 2018, focusing on organization and regulation, funding and service delivery. The following data collection methods were employed: literature review, content analysis of official documents and secondary data, and semi-structured interviews. With regard to organization and regulation, the findings show a lack of institutional mechanisms to mitigate risk selection and that access to private services is intimately linked to ability to pay. The funding model is incapable of sustaining the public health system. With respect to service delivery, despite the implementation of strategies that suggest advances, the segmentation of the system is sustained by the fragmentation of care and purchase of private services. Our findings show that the nature of public-private relations in Chile's health system reinforces the segmentation of population groups produced by the market-oriented approach. Although the reforms implemented during the study period mitigate the effects of segmentation, they were unable to produce structural changes in the configuration of the health system.
\end{abstract}

Key words Health systems, Government regulation, Health system funding, Health care delivery
Resumo $O$ artigo analisa a configuração das relações público-privadas no sistema de saúde do Chile, no período de 2000 a 2018. As dimensões de análise abordaram a organização e a regulação, o financiamento e a prestação de serviços de saúde. Foram utilizadas como técnicas de pesquisa: revisão bibliográfica, análise de documentos $e$ de dados secundários e entrevistas. Três principais achados foram observados. $\mathrm{Na}$ dimensão organizacional e regulatória observou-se escassez de mecanismos institucionais que atenuem a seleção de riscos e vinculação do acesso à capacidade de contribuição dos afiliados do subsistema privado. $O$ modelo de financiamento adotado foi insuficiente para a manutenção do sistema público. Em relação à prestação, apesar da implementação de estratégias que sugerem avanços, a fragmentação do sistema e a compra de serviços privados mantêm a segmentação do sistema. No sistema de saúde chileno, o caráter das relações público-privadas nas dimensões analisadas reitera a segmentação entre grupos populacionais produzidos pela lógica de priorização dos mercados na saúde. As reformas implementadas no período de análise atenuaram os efeitos da segmentação, mas não foram capazes de produzir mudanças estruturais na configuração do sistema de saúde.

Palavras-chave Sistemas de saúde, Regulação governamental, Financiamento dos sistemas de saúde, prestação de assistência à saúde 


\section{Introdução}

A relação entre Estado, mercados e famílias é importante para compreensão dos sistemas de proteção social ${ }^{1}$, cuja constituição se baseia no compromisso estatal de garantir o bem-estar dos cidadãos frente aos riscos inerentes às sociedades mercantis ${ }^{2,3}$. Ao analisar as configurações das políticas sociais, Esping-Andersen ${ }^{1}$ utilizou o conceito de desmercantilização para indicar o grau variável em que as políticas estatais articulam transferências de recursos e serviços sociais públicos, para reduzir a dependência dos cidadãos em relação ao mercado de trabalho.

A América Latina se caracteriza por distintos arranjos de proteção social em saúde ${ }^{4}$. Ao longo do século XX, a intervenção estatal se orientou para implementação de políticas sanitárias voltadas aos trabalhadores formais, com diferenciação dos benefícios, em um sistema do tipo seguro social ocupacional ${ }^{5}$. Contudo, restrições econômicas, desigualdades e ampla informalidade laboral impossibilitaram a inclusão de grande parte da população $0^{6}$.

Nas décadas de 1980 e 1990, as reformas neoliberais na região atingiram os sistemas de proteção social e de saúde ${ }^{7}$. Segundo recomendações de agências internacionais, o Estado deveria se ocupar dos indivíduos e famílias vulneráveis, diminuindo sua atuação por meio da descentralização, focalização e privatização dos programas sociais8.

O Chile, durante décadas expandiu a cobertura do seguro social e caminhou no estabelecimento de um modelo de seguridade social mais universalista e independente do mercado de trabalho, incluindo a criação, nos anos 1950, do Serviço Nacional de Saúde. Em sentido oposto, nos anos 1980, durante a ditadura militar, implantou-se uma reforma neoliberal na saúde baseada no modelo de seguro social com ênfase na participação do mercado 9,10 .

A reforma chilena do período autoritário inaugurou a mais intensa articulação entre Estado e mercado na saúde latino-americana. Estabeleceu-se concorrência por contribuintes entre os subsistemas público e privado, instituiu-se uma transformação na estrutura e funcionamento do sistema de saúde, que passou a se constituir por heterogêneas organizações ${ }^{11}$. $\mathrm{O}$ caso chileno ainda é considerado um exemplo de modelo neoliberal implantado no setor saúde em face do percurso histórico, político e institucional das políticas, das características de articulação entre Estado e mercado e do papel do setor privado configurado desde os anos 1980.
Nos anos 2000, o governo Lagos implementou a reforma do setor saúde a partir de cinco projetos, modificando aspectos relacionados às funções de organização do sistema, regulação do subsistema privado, ampliação de fontes de financiamento e inclusão de novos problemas de saúde na prestação de serviços ${ }^{12}$ que não alteraram a dinâmica do mix público-privado.

$\mathrm{O}$ artigo analisa a configuração das relações público-privadas no sistema de saúde do Chile, de 2000 a 2018, no que concerne às dimensões de organização e regulação, financiamento e prestação de serviços de saúde. Busca-se compreender as características das relações entre Estado e mercados em saúde, discutir suas implicações para a segmentação do sistema e os desafios ao exercício do direito à saúde.

\section{Método}

Trata-se de estudo de caso sobre as relações público-privadas na configuração do sistema de saúde do Chile. Parte-se das contribuições de Immergut ${ }^{13}$ que analisou modalidades de intervenção governamental nas políticas de saúde na Europa, considerando a participação do Estado e dos mercados em quatro dimensões: regulação e organização, financiamento, propriedade dos serviços e emprego em saúde ${ }^{13}$. O estudo considerou três eixos analíticos com base nos estudos da autora: 1) Configuração do sistema quanto a aspectos organizacionais e regulatórios; 2) Relações público-privadas no financiamento e 3) Relações público-privadas na prestação de serviços.

No primeiro eixo, caracterizou-se a estrutura e cobertura do sistema de saúde, definições e atribuições das esferas governamentais, instrumentos de regulação e controle do subsistema privado. No segundo, a composição das fontes de financiamento, gastos públicos e privados em saúde; gastos por desembolso direto; participação da saúde no PIB e copagamentos. No terceiro eixo, enfatizou-se a descrição da oferta de serviços de saúde e participação dos prestadores públicos e privados.

O recorte temporal abrangeu o período de 2000 a 2018. Em 2000 iniciou-se a reforma do setor saúde realizada no Governo Lagos (20002006), com modificações na organização e regulação do sistema, financiamento e prestação de serviços, e, 2018 foi o último ano do segundo governo Bachelet.

As estratégias metodológicas compreenderam: revisão bibliográfica, análise de conteúdo 
de documentos oficiais, dados secundários e entrevistas semiestruturadas.

A análise documental abrangeu documentos governamentais e de órgãos oficiais, incluindo legislação e resoluções do Congresso Nacional, do Tribunal Constitucional e da Presidência da República, documentos técnicos do Ministério da Saúde, Fondo Nacional de Salud (Fonasa), Instituciones de Salud Previsional (Isapre), Superintendencia de Salud e da Comissão Econômica para América Latina e Caribe (CEPAL).

Os dados secundários foram obtidos dos sistemas de informações oficiais do Departamento de Estadísticas e Información de Salud e órgãos relacionados ao Ministério da Saúde, ao Fonasa e às Isapre, bases da Organização para Cooperação e Desenvolvimento Econômico (OCDE), Organização Pan-americana da Saúde (OPS), e Organização Mundial de Saúde (OMS). Complementarmente, utilizaram-se informações de entrevistas semiestruturadas realizadas em 2019 com 14 atores-chave que participaram da política de saúde chilena no período.

A pesquisa foi aprovada pelo Comitê de Ética em Pesquisa da instituição responsável.

\section{Resultados}

\section{Configuração do sistema de saúde: modelo de organização e regulação}

O Chile é um país em desenvolvimento, economicamente estável, com aproximadamente 19 milhões de habitantes. O Estado chileno é unitário e democrático, com administração funcional e territorial descentralizada, dividido em 15 regiões, 54 províncias e 346 comunas.

Apesar da desigualdade social histórica, entre 2013 a 2017, observou-se redução da pobreza ${ }^{11}$, relacionada ao aumento da renda e à expansão de políticas sociais voltadas aos mais vulneráveis.

O país experimentou ganhos na expectativa de vida desde 1970, com aumento de idosos (12\% da população em 2018) e apresentou bom desempenho em indicadores econômicos, sociais e sanitários, em comparação à América Latina (Tabela 1).

O sistema de saúde caracteriza-se por um mix público-privado no financiamento e na prestação de serviços. O marco institucional e a estrutura foram constituídos durante a reforma implementada na ditadura militar no final dos anos 1970. No subsistema público, criou-se o Sistema Nacional de Servicios de Salud (SNSS), composto pelo Ministério da Saúde e organismos dependentes, como o Fonasa, responsável pelo asseguramento público e pela gestão do financiamento das ações de saúde pública. O subsistema privado constituído pelas Isapre, empresas privadas responsáveis pelo asseguramento e prestação de serviços.

Após a reconfiguração do sistema de saúde, estabeleceram-se as principais características do modelo de financiamento, prestação e atenção à saúde. Os trabalhadores optam entre afiliar-se ao Fonasa ou a uma Isapre, por meio da contribuição obrigatória de 7\% do salário. Todavia, a depender da renda e do subsistema devem ser efetuados copagamentos.

Desde sua criação aos dias atuais, o número de afiliados do Fonasa foi se ampliando, constituindo, em 2017, 78,0\% da população ${ }^{17}$, dividida em quatro grupos de acordo com suas condições socioeconômicas e em duas modalidades de atenção à saúde ${ }^{18}$. O grupo $\mathrm{A}$ do Fonasa compreendia usuários sem inserção no mercado formal de trabalho e recursos para contribuições, vinculados à modalidade de atenção institucional (MAI), na qual a assistência médica era realizada em serviços públicos gratuitamente. Os usuários dos grupos B, C e D podiam optar pela modalidade MAI ou pela livre eleição (MLE), que permitia a escolha de profissionais ou serviços do subsistema privado por meio de copagamentos (Quadro 1).

O subsistema privado atendia, em 2017, a $14,4 \%$ da população, sendo constituído por doze Isapre subdivididas em seis abertas (Colmena Golden Cross, Cruz Blanca, Vida Tres, Nueva Masvida, Banmédica, Consalud) que podem ser acessadas pela população em geral e seis fechadas (San Lorenzo, Fusat, Chuquicamata, Río Blanco, Fundación, Cruz del Norte) direcionadas a determinados grupos de trabalhadores ou empresas ${ }^{18}$. As Isapre possuem três modalidades de atenção: plano de livre eleição, plano fechado (o mais econômico) e plano com prestadores preferentes (Quadro 1). A maioria dos afiliados das Isapre (69\%) pertenciam ao grupo com renda igual ou superior a US\$ 1.1000 mensais, viviam na região metropolitana do Chile $(59,3 \%)$, na maioria homens $(63,4 \%)$, com menos de 40 anos $(47,4 \%)^{19}$.

Com relação à reitoria e gestão do sistema, em 2005, a Lei de Autoridade Sanitária e Gestão reorganizou as funções do Ministério da Saúde e instituições relacionadas (Quadro 1), cujo objetivo foi promover seu fortalecimento institucional pela ampliação das atribuições ${ }^{20}$. Além disso, foram constituídas a Subsecretaria de Redes Asistenciales e a Subsecretaria de Salud Pública.

No nível regional dividiram-se as funções de fiscalização e regulação (a cargo das Secretarias 
Tabela 1. Indicadores básicos selecionados do Chile e média da América Latina, 2016, 2019.

\begin{tabular}{|c|c|c|}
\hline Indicadores & Chile & América Latina \\
\hline População total (em milhões) (2019) & 18.952 .000 & 640.463 .000 \\
\hline PIB per capita em ppp (US\$) (2018) & 22.874 & $14.428^{\mathrm{a}}$ \\
\hline Crescimento anual do PIB (2018) & $4,0 \%$ & $1,4 \%$ \\
\hline Coeficiente de Gini (2018) & 0,45 & 0,46 \\
\hline Expectativa de vida ao nascer (Total) (2019) & 80,2 & 75,6 \\
\hline Homens & 77,8 & 72,5 \\
\hline Mulheres & 82,4 & 78,8 \\
\hline Porcentagem de população $\geq 65$ anos (2019) & $12 \%$ & $9 \%$ \\
\hline Média de anos de escolaridade (Total) & $10,3(2015)$ & $8,6(2016)$ \\
\hline Homens & 10,5 & 8,5 \\
\hline Mulheres & 10,2 & 8,6 \\
\hline Partos hospitalares (\%) (2017) & $99,6 \%$ & $92,4 \%$ \\
\hline Razão de Mortalidade materna notificada/100 mil nascidos vivos (2017) & 17,3 & 69,3 \\
\hline Taxa de Mortalidade infantil notificada/1000 nascidos-vivos (2017) & 7,1 & 14,8 \\
\hline Taxa de mortalidade neonatal notificada/1000 nascidos-vivos (2017) & 5,5 & 9,3 \\
\hline Mortalidade por câncer de próstata ${ }^{\mathrm{b}}$ (100 mil habitantes) (2016) & 24,2 & 20,4 \\
\hline Mortalidade por câncer de mama ${ }^{\mathrm{b}}$ (100 mil habitantes) (2016) & 13,4 & 14,3 \\
\hline Mortalidade por acidente vascular cerebral ${ }^{\mathrm{b}}$ (100 mil habitantes) (2016) & 37,3 & 43,4 \\
\hline $\begin{array}{l}\text { Mortalidade por enfermidades isquêmicas do coração }{ }^{\mathrm{b}}(100 \mathrm{mil} \\
\text { habitantes) (2016) }\end{array}$ & 48,4 & 87,8 \\
\hline
\end{tabular}

Regionales Ministeriales) e de provisão (a cargo dos Servicios de Salud) e organizou-se o modelo de atenção em redes assistenciais. No nível local, os Departamentos de Salud das comunas eram responsáveis pela administração dos estabelecimentos de atenção primária à saúde (APS), sob diretrizes da Subsecretaria de Redes Asistenciales.

A Superintendencia de Salud é responsável pela regulação e fiscalização dos prestadores públicos e privados quanto à acreditação e certificação dos estabelecimentos de saúde, definição de garantias para pagamento dos serviços dos prestadores de saúde, cumprimento da legislação sobre direitos e deveres das pessoas no setor saú$\mathrm{de}^{21}$ (Quadro 1).

Em 2005, no bojo da reforma da saúde, a Superintendencia de Salud substituiu a Superintendencia de Isapre. Ademais, este órgão ficou responsável pela regulação e fiscalização do Fonasa e Isapre para o cumprimento das Garantias Explícitas de Saúde (GES). O GES foi a principal estratégia de reforma e consistiu em garantir, para os setores público e privado, prestações de saúde definidas para uma lista de patologias.

Entretanto, persistiram entraves quanto à regulação do subsistema privado, especialmente no que se refere ao controle dos preços dos planos.
Em 2010, o Tribunal Constitucional chileno declarou a inconstitucionalidade do texto que estabelecia a competência da Superintendencia de Salud de fixar a fatores de risco por sexo e idade ${ }^{22}$. A decisão se baseou na falta de racionalidade na distinção por idade e sexo, violação do direito à proteção da saúde e à segurança social.

\section{Relações público-privadas no financiamento}

O financiamento do sistema de saúde chileno é constituído por diversas fontes, diretas e indiretas, dos subsistemas público e privado como: impostos gerais e específicos, recursos orçamentários, contribuições compulsórias e voluntárias e copagamentos. (Quadro 2)

O gasto em saúde aumentou de 2000 a 2018 como proporção do PIB sendo que os gastos públicos superaram os privados no período ${ }^{20}$. Entretanto, apesar do aumento progressivo, o gasto em saúde per capita público e privado é um dos menores da OCDE, superior somente aos do México e Turquia, assim como o gasto público per capita que ocupa uma das últimas posições ${ }^{16}$ (Gráfico 1). 
Quadro 1. Estrutura político-administrativa e aspectos organizacionais do sistema de saúde chileno, 2020.

\begin{tabular}{|l|l|}
\hline \multicolumn{1}{|c|}{ Dimensões } & \multicolumn{1}{c|}{ Descrição } \\
\hline $\begin{array}{l}\text { Estrutura político- } \\
\text { administrativa }\end{array}$ & Estado Unitário - 15 regiões, 54 províncias e 346 comunas \\
\hline $\begin{array}{l}\text { Marco } \\
\text { constitucional da } \\
\text { saúde }\end{array}$ & $\begin{array}{l}1980 \text { - Constituição da República - Artigo 19 No 9 Dever do Estado como garantidor } \\
\text { da execução das ações de saúde, prestadas por instituições públicas ou privadas. } \\
\text { Possibilidade de contribuições obrigatórias e direito do cidadão afiliar-se ao sistema } \\
\text { público ou privado. }\end{array}$ \\
\hline $\begin{array}{l}\text { Modelo de } \\
\text { proteção social } \\
\text { e estrutura do } \\
\text { sistema de saúde }\end{array}$ & $\begin{array}{l}\text { Modelo dual } \\
\text { Público } \\
\text { Fssistencial - Cobertura parcial para pobres e pessoas sem acesso ao seguro social. } \\
\text { Seguro social - Cobertura parcial para trabalhadores contribuintes e beneficiários e } \\
\text { copagamentos. Fonasa B, C e D }\end{array}$ \\
& $\begin{array}{l}\text { Privado } \\
\text { Seguro social - Cobertura parcial para trabalhadores contribuintes e beneficiários, } \\
\text { contribuições adicionais voluntárias e copagamentos. }\end{array}$ \\
\hline $\begin{array}{l}\text { Fonasa - 78\% da população (13.926,475 afiliados) } \\
\text { A -23,71\%; B - 33,00\%; C - 19,05\%; D - 24,21\% }\end{array}$ \\
$\begin{array}{l}\text { Isapre - 14,4\% da população (1.628.152 afiliados) } \\
\text { Doze Isapre: } \\
\text { Abertas - 97,1 \% } \\
\text { Fechadas - 2,2\% } \\
\text { Outros - 7,6\% } \\
\text { Forças Armadas e de Ordem - 2,8\% } \\
\text { Naro informado-2,0\% }\end{array}$ \\
\hline 2017$)$
\end{tabular}

continua

No Fonasa, em 2018, os gastos em saúde foram financiados em sua maioria por impostos gerais $(73 \%)$ e pela contribuição obrigatória $(27 \%)$. Observa-se a presença crescente do gasto público advindo de impostos gerais para o financiamento do gasto público em saúde ao comparar 2005 (42\%), 2010 (57\%) e 2015 (59\%). A reestruturação dos gastos públicos permitiu o aumento do investimento nas áreas sociais e na saúde, com crescimento da participação no orçamento governamental.

Nas Isapre os gastos em saúde foram provenientes da contribuição obrigatória $(72 \%)$ e das adicionais voluntárias $(28 \%)^{23}$. De 2012 a 2018, das doze Isapre, nove apresentaram lucro.

Os copagamentos de prestações de saúde e medicamentos correspondiam a um importante componente dos gastos privados em saúde das famílias. Apesar do decréscimo entre 2000 e 2018 de $42,8 \%$ para $35,1 \%$, o Chile apresentava gastos por desembolso direto bem superiores à média dos países da OCDE, ficando atrás apenas do México $^{16}$.

No subsistema público, o pagamento a prestadores da APS na modalidade institucional é majoritariamente per capita por afiliados inscritos nos centros de saúde e, em menor proporção, por serviço ${ }^{17}$. Já na atenção secundária e terciária se combinam pagamentos por serviços e projeção histórica das prestações de saúde nos hospitais. A modalidade de livre eleição do Fonasa e o subsistema privado realizam pagamentos por serviço pós-prestação (Quadro 2).

Por fim, as transferências de recursos públicos, relacionadas à modalidade de livre eleição e ao GES, ao subsistema privado variaram de $3,82 \%$ em 2012 para 3,92\% em 2017. Neste intervalo, a porcentagem mais alta dos gastos públicos transferidos para o subsistema privado foi de $4,12 \%$, em $2016^{27}$. 
Quadro 1. Estrutura político-administrativa e aspectos organizacionais do sistema de saúde chileno, 2020.

\begin{tabular}{|c|c|}
\hline Dimensões & Descrição \\
\hline $\begin{array}{l}\text { Modalidades de } \\
\text { atenção }\end{array}$ & $\begin{array}{l}\text { Fonasa - Plano único com cesta de prestações em duas modalidades. } \\
\text { Modalidade de atenção institucional (MAI) - atendimento em instituições públicas e } \\
\text { centros de saúde de APS. } \\
\text { Atenção gratuita - Grupos A, B e maiores de } 60 \text { anos } \\
\text { Cobertura de } 90 \% \text { - Grupo C } \\
\text { Cobertura de } 80 \% \text { - Grupo D } \\
\text { Modalidade de livre eleição - grupos B, C e D - pagamento de vouchers em } \\
\text { estabelecimentos públicos ou privados. Cobertura menor e copagamento maior. } \\
\text { Isapre - diferentes modelos de atenção, bonificações, cobertura e prestadores, nas } \\
\text { modalidades: } \\
\text { Plano de livre eleição -afiliado escolhe no mercado, e quita copagamentos de acordo com a } \\
\text { cobertura pactuada. } \\
\text { Plano fechado -afiliado utiliza somente alguns prestadores. } \\
\text { Plano com prestadores preferentes -afiliado atendido nos prestadores preferentes do plano, } \\
\text { com copagamento menor, ou em prestador de sua escolha, com copagamento maior. }\end{array}$ \\
\hline $\begin{array}{l}\text { Estrutura e } \\
\text { organização do } \\
\text { sistema de saúde }\end{array}$ & $\begin{array}{l}\text { Nacional: } \\
\text { Ministério da Saúde: aspectos normativos, regulatórios e gestão nacional. } \\
\text { Subsecretaria de Redes: normatização da articulação e desenvolvimento da rede } \\
\text { assistencial e regulação das prestações de saúde. } \\
\text { Subsecretaria de Salud Pública: funções reguladoras, normativas, de vigilância e } \\
\text { fiscalizadoras de promoção da saúde, prevenção e controle de enfermidades. } \\
\text { Superintendencia de Salud: regulação e fiscalização dos seguros e prestadores de saúde } \\
\text { pública e privada, preservação de direitos, promoção da qualidade e segurança na atenção. } \\
\text { Instituto de Salud Pública: avaliação dos laboratórios, vigilância de enfermidades, controle } \\
\text { de medicamentos, cosméticos e dispositivos médicos, saúde mental, ocupacional, } \\
\text { produção e controle da qualidade de vacinas. } \\
\text { Regional: } \\
\text { Secretarias Regionales Ministeriales de Salud: cumprimento das normas, planos, e políticas } \\
\text { nacionais, adequando-os à realidade regional. } \\
\text { Servicios Regionales de Salud: articulação, gestão e desenvolvimento da rede assistencial } \\
\text { para execução das ações de proteção, recuperação, reabilitação e cuidados paliativos. } \\
\text { Local: } \\
\text { Departamentos de Salud municipais: prestação de serviços de APS; administração de } \\
\text { centros de saúde das comunas. }\end{array}$ \\
\hline $\begin{array}{l}\text { Regulação e } \\
\text { controle do } \\
\text { subsistema privado }\end{array}$ & $\begin{array}{l}\text { Superintendencia de Salud: } \\
\text { - Fiscalização e controle das Isapre, Fonasa e regime de Garantias Explícitas em Saúde. }\end{array}$ \\
\hline
\end{tabular}

Fonte: Elaboração própria, diversas fontes. 
Quadro 2. Características do financiamento em saúde no Chile, 2018.

\begin{tabular}{|c|c|}
\hline Dimensões & Descrição \\
\hline $\begin{array}{l}\text { Indicadores de } \\
\text { gasto selecionados }\end{array}$ & $\begin{array}{l}\text { Gasto em saúde } \% \text { do PIB: } 8,9 \% \\
\text { Gasto público como } \% \text { do gasto em saúde: } 58,3 \% \\
\text { Desembolso direto como \% do gasto em saúde: } 35,1 \% \\
\text { Gasto em saúde financiado pelo Fonasa a Isaprea: } 3,9 \% \text { (2017) }\end{array}$ \\
\hline $\begin{array}{l}\text { Fontes de } \\
\text { financiamento }\end{array}$ & $\begin{array}{l}\text { Público } \\
\text { Orçamento Geral (impostos) } \\
\text { Recursos dos Ministérios da Saúde e da Defesa } \\
\text { Recursos municipais (APS) } \\
\text { Contribuições do seguro social obrigatório dos trabalhadores e empresas (7\% do salário) } \\
\text { Privado } \\
\text { Desembolso direto: copagamentos atenção médica, medicamentos e consultas } \\
\text { particulares } \\
\text { Contribuições do seguro social obrigatório dos trabalhadores e empresas (7\% do salário) } \\
\text { Contribuições adicionais do seguro privado }\end{array}$ \\
\hline $\begin{array}{l}\text { Modalidades } \\
\text { de pagamento a } \\
\text { prestadores }\end{array}$ & $\begin{array}{l}\text { Fonasa } \\
\text { Provedores públicos - MAI } \\
\text { APS municipal - Pagamento per capita e por serviço } \\
\text { APS dependentes dos Servicios de Salud - Pagamento por serviço; orçamento global, } \\
\text { projeção histórica das prestações e por programas } \\
\text { Atenção secundária e terciária - Orçamento global e projeção histórica das prestações; } \\
\text { pagamento por serviço } \\
\text { Provedores privados - MAI } \\
\text { Atenção secundária e terciária: pagamento por serviço; pagamento por caso } \\
\text { Provedores privados - MLE } \\
\text { Atenção secundária e terciária: pagamento por serviço } \\
\text { Isapre } \\
\text { Atenção primária, secundária e terciária: pagamento por serviço }\end{array}$ \\
\hline
\end{tabular}

${ }^{a}$ Transferências realizadas de três principais programas: compra de serviços pelo Fonasa ou Servicios Regionales de Salud; Modalidade de livre eleição; Bônus GES.

Fonte: OCDE, 2019 ${ }^{16}$; Fonasa, 2018 ${ }^{17}$; Minsal, 201920; Clínicas de Chile, 2016 ${ }^{24}$; Superintendencia de Salud, $2020^{25}$.

Em 2017, as compras de prestações não GES estavam relacionadas principalmente a leitos críticos, serviços de hemodiálise e serviços de urgência. As compras GES eram relativas a serviços oncológicos e bônus GES, estratégia iniciada em 2010 que garantiu aos beneficiários do Fonasa o atendimento das patologias GES em estabelecimentos privados ${ }^{27}$.

\section{Relações público-privadas na prestação de serviços}

A prestação de serviços ambulatoriais e especializados é realizada por estabelecimentos públicos e privados. Em 2005, o governo do presidente Lagos promoveu mudança no modelo de APS com a implantação do Modelo de Atención
Integral de Salud Familiar y Comunitária e implementação da Rede Integrada de Serviços de Saúde, a partir de 2014 .

Os estabelecimentos públicos de APS são constituídos por Centros Comunitarios de Salud Familiar (CECOSF) (9,1\%); Centros de Salud Familiar (CESFAM) (19,3\%); Centro de Salud Urbano (3,9\%); Centro de Salud Rural (2,9\%); Posta de Salud Rural (52,7\%) e Servicio de Atención Primaria de Urgencia (SAPU) (12,1\%) (Quadro 3). Após a reforma, investiu-se na infraestrutura do subsistema público com a construção de hospitais e dos CECOSF, unidades de APS próximas à comunidade, complementares aos CESFAM.

A atenção ambulatorial no subsistema privado e os estabelecimentos que atendem a população inscrita na modalidade de livre eleição são 


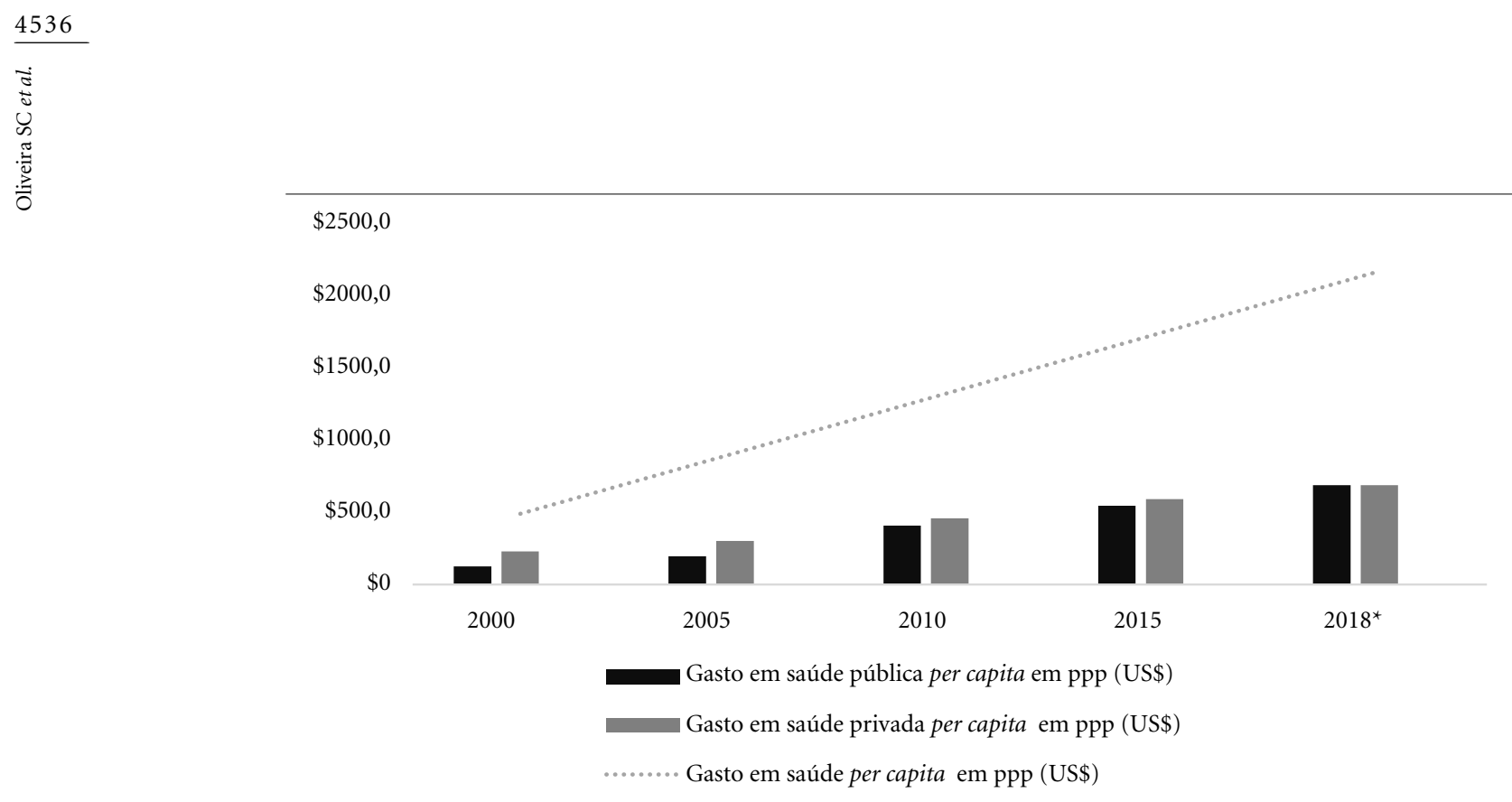

Gráfico 1. Evolução do gasto total em saúde e composição dos gastos público e privado, de 2000 a 2018.

* Dados relativos aos gastos públicos, privados e desembolso direto são referentes ao ano de 2017.

Fonte: WHO, $2020^{26}$.

constituídos por 2.344 estabelecimentos de saúde divididos em Centros Médicos (31,8\%); Centros de Diálise (0,7\%); Clínicas Cirúrgicas (9,2\%); Laboratórios $(37,4 \%)$ e Centros de Imagem (20,9\%) (Quadro 3).

A oferta da atenção secundária, no subsistema público, é organizada pelos Servicios de Salud de forma regionalizada, sendo responsável pela articulação, gestão e desenvolvimento da Rede Assistencial. As direções dos Servicios de Salud são responsáveis pela integração da rede ${ }^{28}$. Entre os hospitais prestadores do Fonasa destacam-se os Centros de Diagnostico Terapéutico (CDT), Centros de Salud Mental (COSAM) e Centros de Referencia de Salud (CRS). Os agendamentos são realizados conforme referência da APS.

O Chile aumentou a densidade de médicos, mas ainda está abaixo da média dos países desenvolvidos. Em 2018, esse indicador alcançava 2,6 médicos por 1000 habitantes, menor que a média da $\operatorname{OCDE}(3,4$ em 2017). As consultas médicas aumentaram de 2,4 para 3,6 per capita entre 2000 e 2016 .

Dos hospitais (baixa, média e alta complexidade), 54,6\% são públicos e $23,9 \%$ privados. Associações de mútua ajuda e estabelecimentos institucionais representam 3,2\% e 4,6\% dos hospitais. Os leitos são majoritariamente públicos (68\% do total). O subsistema privado é responsável por $18 \%$ dos leitos hospitalares em clínicas privadas, $9 \%$ nos estabelecimentos institucionais e $5 \%$ em outros estabelecimentos. No período analisado houve pequeno decréscimo no número de leitos e, especificamente nos leitos psiquiátricos, que foram reduzidos pela metade, em função do processo de reforma psiquiátrica desde a década de 1990. A taxa de internação teve um aumento de 5,8 para 6,0 dias no período entre 2000 e 2017.

Em relação ao apoio à diagnose e terapia, entre 2011 e 2017 houve aumento na oferta de ressonância magnética (de 4,1 para 12,3 por 1 milhão de habitantes) e tomografia computadorizada (de 10,2 para 24,3 por 1 milhão de habitantes).

O Quadro 3 sintetiza as principais características da prestação de serviços do sistema de saúde.

\section{Discussão}

A análise das relações público privadas em saúde no Chile evidencia persistente segmentação na configuração do sistema. As contradições do modelo voltado ao mercado, colocam três desafios principais ao sistema de saúde.

$\mathrm{O}$ primeiro, relaciona-se à organização e regulação do sistema de saúde, que não desenvolveu mecanismos para reduzir a seleção de riscos, 
Quadro 3. Características da prestação de serviços no sistema de saúde do Chile, de 2000 a 2018.

\begin{tabular}{|c|c|}
\hline Componentes & Descrição \\
\hline $\begin{array}{l}\text { APS, Atenção ambulatorial } \\
\text { e secundária }\end{array}$ & $\begin{array}{l}\text { Modelo de Atenção Integral à Saúde Familiar e Comunitária territorializado, com } \\
\text { inscrição dos usuários nas unidades de APS. } \\
\text { Equipe: médico, enfermeiro, matrona, assistente social, assistente administrativo, } \\
\text { agente comunitário } \\
\text { Vínculo e remuneração: funcionários públicos (maioria) e contratos temporários; } \\
\text { salário + pagamento por desempenho (programas específicos). } \\
\text { Atenção secundária: derivação das unidades de APS mediante documento de } \\
\text { interconsulta; consultas médicas; procedimentos; exames; medicamentos e } \\
\text { cirurgias sem hospitalização } \\
\text { Atenção ambulatorial (2016) } \\
\text { Pública } \\
\text { Total: } 2 \text { 238 } \\
\text { Centro Comunitário Salud Familiar - 9,1\% } \\
\text { CESFAM - 19,3\% } \\
\text { Centro de Salud Urbano - 3,9\% } \\
\text { Centro de Salud Rural - 2,9\% } \\
\text { Posta de Salud Rural - 52,7\% } \\
\text { SAPU - 12,1\% } \\
\text { Atenção secundária pública } \\
\text { Total: } 109 \\
\text { Centro de Referencia de Salud (CRS) - 9,2\% } \\
\text { Centro de Diagnóstico Terapéutico (CDT) - 13,8\% } \\
\text { Centros de Salud Mental (COSAM) - 77,0\% } \\
\text { Atenção ambulatorial e secundária privada (Modalidade livre eleição) } \\
\text { Centros Médicos - } 31,8 \% \\
\text { Centros de Diálise - } 0,7 \% \\
\text { Clínicas Cirúrgicas - 9,2\% } \\
\text { Laboratórios - 37,4\% } \\
\text { Centros de Imagem - 20,9\% }\end{array}$ \\
\hline Hospitais (2016) & $\begin{array}{l}\text { Estabelecimentos: } 348 \\
\text { Públicos - 54,9\% (baixa complexidade -101; média complexidade -24; alta } \\
\text { complexidade - 63). } \\
\text { Privados - } 23,9 \% \\
\text { Associações de ajuda mútua - 3,2\% } \\
\text { Psiquiátricos, geriatria, recuperação - 10,9\% } \\
\text { Institucionais (Forças Armadas, universitários) - } 4,6 \% \\
\text { Outros - } 2,5 \% \\
\text { Leitos / } 1000 \text { hab (2017): } 2,1 \\
\text { Leitos: } 38362 \\
\text { Públicos - } 68 \% \\
\text { Privados - } 18 \% \\
\text { Mutuales - } 2 \% \\
\text { Clínicas psiquiátricas e de geriatria - } 3 \% \\
\text { Institucionais (Forças Armadas, universitários) - } 9 \%\end{array}$ \\
\hline
\end{tabular}

Fonte: OCDE, $2019^{16}$ Fonasa, $2018^{17}$; Clínicas de Chile, $2016^{24}$.

especialmente relacionados à idade e sexo, entre os afiliados do Fonasa e Isapre ${ }^{11}$. Da criação das Isapre até a retomada da democracia não havia regulação, fiscalização ou controle das operações do subsistema privado. As Isapre puderam classificar e selecionar os riscos cobertos por apro- 
ximadamente dez anos, sem intervenção governamental ${ }^{29}$.

No subsistema privado, além da cotização obrigatória, há outras contribuições pagas de acordo com a cobertura e o plano. A cobertura de serviços está intimamente ligada à capacidade de contribuição do afiliado ou do grupo familiar, visto ser possível rejeitar a afiliação a partir de mecanismos de seleção de riscos baseado em informações socioeconômicas e do histórico fami$\operatorname{liar}^{30}$. Em contrapartida, o sistema público atende no Fonasa A e B mais de $50 \%$ da população em situação de vulnerabilidade e os mais pobres.

Embora tenham sido realizadas mudanças organizacionais como reestruturação e separação das funções do Ministério da Saúde e órgãos subordinados, ampliação das funções de fiscalização e regulação da Superintendencia de Salud na reforma de 2005, os subsistemas público e privado permanecem com escassez de mecanismos organizacionais que atenuem essas distorções do sistema de seguro social. Os mecanismos regulatórios criados após a reforma do governo Lagos não enfrentaram o problema, persistindo as seleções por doenças pré-existentes; aumentos periódicos dos prêmios; variação de copagamentos e benefícios (por mudança de planos); prêmios ajustados por riscos etc $^{31}$. A manutenção desta organização pode causar restrições no subsistema público devido ao aumento crescente dos gastos e escassez de recursos.

O segundo desafio se refere ao modelo de financiamento. A contribuição obrigatória destinada ao Fonasa não possibilita suficiência do financiamento. Os recursos de impostos gerais obtiveram um progressivo crescimento no período de análise, alcançando mais de 70\% do financiamento em 2018. Em contrapartida, 75\% das Isapre obtiveram lucro nos últimos anos. Apesar de questionamentos quanto às contribuições obrigatórias direcionadas ao subsistema privado e mudanças legislativas, a estrutura do sistema de saúde não logrou alteração mais ampla ${ }^{32}$.

No período de análise, apesar do incremento progressivo, o Chile manteve uma das menores participações públicas no gasto em saúde e um dos menores gasto per capita da $\mathrm{OCDE}^{16}$. O gasto público como porcentagem do PIB $(4,8 \%$ em 2016) se manteve abaixo das recomendações da OMS de 6\% para os países em desenvolvimento das Américas ${ }^{33}$.

A persistência deste modelo gerou consequências negativas como desigualdades no acesso, cobertura, qualidade da atenção e no financiamento, pois apesar do aumento no gasto público em saúde nos últimos anos, corresponderam a somente $50 \%$ dos gastos totais, para cobrir $78 \%$ da população ${ }^{30}$.

A população chilena apresenta expressivo gasto por desembolso direto (31,1\% em 2016), principalmente com a compra de medicamentos e copagamentos, em comparação à média da OCDE ou da América Latina ${ }^{34,15}$. A baixa cobertura financeira frente aos gastos catastróficos desvela um sistema desigual e injusto, principalmente para a população mais vulnerável ${ }^{30}$.

A prestação em saúde se caracteriza como o terceiro desafio do sistema chileno. Houve avanços na garantia do acesso à assistência médica com a implantação do Modelo de Atención Integral de Salud Familiar y Comunitária e a integração dos serviços de saúde em rede. Entretanto, apesar de serem estratégias para orientação do cuidado no subsistema público de saúde, não houve integração com o subsistema privado ${ }^{28}$. Na atenção hospitalar, o subsistema público detém a maioria dos leitos, conformando uma sólida estrutura pública, com ampliação dos serviços de apoio à diagnose e terapia, apesar da dependência da compra de prestações privadas.

Outro importante avanço foi o aumento progressivo das patologias GES entre 2005 e 2013. Porém, não ocorreu o ajuste das contribuições obrigatórias entre Fonasa e Isapre, persistindo a alocação desigual dos recursos ${ }^{35}$. Ao contrário, para atender à legislação houve necessidade de compra de prestações relacionadas ao regime de garantias, principalmente referentes ao tratamento de prestações oncológicas e bônus GES, estratégia criada para diminuir as filas de espera $\mathrm{GES}^{27}$.

A implementação do regime estabeleceu um novo tipo de fragmentação do cuidado que favoreceu ações curativas, e reiterou a segmentação do sistema, já que os usuários com patologia GES têm garantia legal de atenção e os não-GES não ${ }^{36}$.

\section{Conclusão}

O Chile é um caso paradigmático de priorização dos mercados em saúde, por meio de relações público-privadas na regulação, financiamento e prestação de serviços que reiteram a segmentação entre grupos populacionais.

Destaca-se que mesmo em meio à crise político-institucional originada por protestos populares a partir de outubro de 2019, o governo chileno apresentou, no início de 2020, uma proposta de cobertura universal dos serviços de saú- 
de do subsistema público, com pacote definido de benefícios ${ }^{37}$. Buscou-se materializar o projeto incentivado por agências internacionais para países de renda média e baixa, cuja ênfase tem sido o papel subsidiário do Estado na atenção à saúde e ampliação das relações com o mercado ${ }^{38}$.

Além dos problemas oriundos da articulação entre Fonasa e Isapre não terem sido enfrentados, a proposta de cobertura universal pode perpetuar a discriminação de pessoas com maiores riscos e acentuar as iniquidades em saúde. Cabe ao governo e ao Legislativo debaterem uma proposta de reforma estrutural que reconfigure as relações público-privadas, introduza mecanismos de solidariedade entre os subsistemas, diminua a segmentação (de cobertura e financiamento) e a fragmentação entre níveis de atenção.

A reflexão sobre a experiência chilena é relevante para compreender os efeitos de arranjos segmentados e com forte participação privada em países desiguais, como os da América Latina. Estudos comparados de sistemas de saúde latino-americanos com foco em diferentes modelos de intervenção estatal e relações público privadas são necessários, visando subsidiar a construção de sistemas de saúde efetivos e equitativos, que contribuam para a expansão dos direitos e redução das desigualdades sociais.

\section{Colaboradores}

SC Oliveira foi responsável pela realização do trabalho de campo e pela concepção, revisão bibliográfica, redação e aprovação da versão final. CV Machado e PF Almeida participaram da concepção, redação, revisão crítica e aprovação da versão final. ARA Hein participou da revisão crítica e aprovação da versão final.

\section{Agradecimentos}

SC Oliveira foi bolsista da Coordenação de Aperfeiçoamento de Pessoal de Nível Superior (Capes) e edital do Proex-Capes-ENSP 2018. CV Machado e PF Almeida são Bolsistas de Produtividade em Pesquisa do Conselho Nacional de Desenvolvimento Científico e Tecnológico (CNPq). CV Machado é apoiada pela Fundação de Amparo à Pesquisa do Estado do Rio de Janeiro (Faperj) no âmbito do Edital de Apoio a Grupos Emergentes no Estado do Rio de Janeiro.

\section{Referências}

1. Esping-Andersen G. As três economias políticas do Welfare State. Lua Nova 1991; 24:85-116.

2. Titmuss RM. 'What is Social Policy?' In: Abel-Smith B, Titmuss K, editors. Social policy: an introduction, Chapter 2, New York, NY: Pantheon Books, A Division of Random House; 1974. p. 23-32.

3. Offe C. Contradicciones em el estado de bien estar. Madrid: Ediciones Alianza Universidad; 1993.

4. Draibe SM, Riesco M. Estados de bem-estar social e estratégias de desenvolvimento na Amé- rica Latina. Um novo desenvolvimentismo em gestação? Sociologias 2011; 13(27):220-254

5. Haggard S, Kaufman RR. Introduction. Toward a Political Economy of Social Policy. In: Development, democracy and Welfare States: Latin America, East Asia, and Eastern Europe. Princeton: Princeton University Press; 2008. p. 1-24.

6. Mesa-Lago C. As reformas de previdência na América Latina e seus impactos nos princípios de seguridade social. Brasília: Ministério da Previdência Social; 2006.

7. Cantu R. Depois das reformas: os regimes de proteção social latino-americanos na década de 2000. Revista de Sociologia e Politica 2015; 23(56).

8. Almeida C. Reforma de Sistemas de Saúde: tendências internacionais, modelos e resultados. In: Giovanella L, Escorel S, Lobato LVC, Noronha JC, Carvalho AI, organizadores. Políticas e sistema de saúde no Brasil. $2^{a}$ edição. Rio de Janeiro: Editora Fiocruz; 2012. p. 759-802. 
9. Bustos CAM. Institucionalidad sanitária chilena: 1889-1989. Santiago de Chile: LOM Ediciones; 2010.

10. Luzuriaga MJ. Privados de la salud: las políticas de privatización de los sistemas de salud en Argentina, Brasil, Chile y Colombia. 1a edição. São Paulo: Hucitec; 2018.

11. Gattini $\mathrm{CH}$, Arteaga O. Teoría y práctica de aseguramiento privado de salud en Chile: brechas, consistencia y reforma pendiente. Tempus 2016; 10(1):29-42.

12. Bastías G, Valdívia G. Reforma de salud en Chile; El plan AUGE o Régimen de Garantías Explícitas en Salud (GES). Su origen y evolución. Boletín Escuela de Medicina 2007; 32(2) U.C.

13. Immergut E. Health politics: interests and institutions in Western Europe. Cambridge: Cambridge University Press; 1992.

14. Comisión Económica para América Latina y el Caribe. Panorama Social de América Latina, 2019 LC/ PUB.2019/3-P. Santiago de Chile; 2019.

15. Organización Panamericana de la Salud (OPS). Indicadores básicos 2019: Tendencias de la salud en las Américas. Washington, D.C.: OPS; 2019.

16. Organisation for Economic Cooperation and Development (OCDE). Health at a glance 2019: OECD indicators. Paris: OECD Publishing; 2019.

17. Fondo Nacional de Salud (Fonasa). Boletín Estadístico 2016-2017. Santiago de Chile: Fonasa; 2018. [acceso 2020 maio 20]. Disponible en: https://www.fonasa.cl/ sites/fonasa/documentos.

18. Encuesta de Caracterización Socioeconómica Nacional (Encuesta Casen). Observatorio Social. Previsión social: síntesis de resultados. Santiago de Chile: Encuesta Casen; 2017.

19. Superintendencia de Salud. Documento de Trabajo Resultados Financieros del Sistema Isapre: a Diciembre de 2018. Departamento de Estudios y Desarrollo. Elaborado por: Raúl Poblete. Santiago de Chile, 2019. [acceso 2020 Maio 25]. Disponible en: http:// www.supersalud.gob.cl/documentacion/666/articles-18492_recurso_1.pdf.

20. Chile. Ministerio de Salud (MS). Información Económica de Salud. Santiago de Chile: MS; 2019. [acceso 2020 jun 13]. Disponible en: http://ies.minsal.cl/ cuentas/publico/reportel.

21. Chile. Ministério da Saúde (MS). Subsecretaria de Saúde Pública. Lei $n^{\circ}$ 20.584. Regula os direitos e deveres que tem as pessoas em relação às ações vinculadas a sua atenção em saúde. Chile: MS, 2012. [acesso 2020 jun 14]. Disponível em: https://www.leychile.cl/Navegar?idNorma $=1039348$.

22. Chile. Tribunal Constitucional. Sentença no 1710-10, processo iniciado de oficio para decidir sobre a constitucionalidade do artigo 38 da Lei de Isapres. Santiago de Chile; 2010.

23. Instituciones de Salud Previsional (Isapres). Gasto y Financiamiento de la Salud en Chile y en Isapres. Composición del Gasto en Prestaciones y Licencias Médicas en Isapre 2012-2017. Santiago de Chile: Isapres; 2018.

24. Clínicas de Chile. Dimensionamiento del sector de salud privado en Chile. Actualización a cifras año 2016. Santiago de Chile, Clínicas de Chile A.G.; 2016. 190 p.

25. Superintendencia de Salud. Estadísticas Prestadores de Salud. [acceso 2020 jul 25]. Disponible en: http:// www.supersalud.gob.cl/documentacion/666/w3-article-18219.html.
26. World Health Organization (WHO). Global Health Expenditure Database. [cited 2020 Jul 12]. Available from: https://apps.who.int/nha/database/ViewData/ Indicators/en.

27. Dirección de Presupuestos (Dipres). Gobierno de Chile. Ministerio de Hacienda. Santiago de Chile; 2017.

28. Almeida PF, Oliveira SC, Giovanella L. Integração de rede e coordenação do cuidado: o caso do sistema de saúde do Chile. Cien Saude Colet 2018; 23(7):22132228.

29. Escobar L, Bitrán R. Chile: las Garantías Explícitas de Salud. In: Gidion U, Britán R, Tristao I, editors. Planes de Beneficios en salud en América Latina. Banco Interamericano de Desarrollo, Washington, DC; 2014.

30. Organização de Cooperação e de Desenvolvimento Económico (OCDE). Estudios económicos: Chile 2007; 20 (Supl. 2).

31. Cid C. Problemas y desafios del seguro de salud y su financiamiento en Chile: el cuestionamiento a las ISAPRE y la solución funcional. Centro de Políticas Públicas, Pontificia Universidad Católica de Chile 2011; 6(49).

32. Cid C, L Prieto. Gasto de bolsillo en salud: el caso de Chile 1997 y 2007. Rev Panam Salud Publica 2012; 31(4):310-316.

33. Organización Mundial de la Salud (OMS). Financiación de los Sistemas de Salud: El camino hacia la cobertura universal. Informe sobre la salud en el mundo. Suiza: OMS; 2010.

34. Inostroza M, Sánchez H, editores. Construcción Política del Sistema de Salud Chileno: la importancia de la estrategia y la transición. Universidad Andrés Bello: Santiago de Chile; 2017.

35. Huber E, Pribble J, Stephens J. The Chilean left in Power: achievements, failures, and omis-sions. In: Weyland, Kurt; Madrid, Raúl; Hunter, Wendy. Leftist governments in Latin American: successes and shortcomings. Cambridge; 2010.

36. Parada M, Reyes C, Cuevas K, Ávila A, López P, Carrasco V, et al. Transformaciones del Sistema de Salud Público post Reforma AUGE-GES en Valparaíso. Rev Chil Salud Publica 2014; 18(2):127-139.

37. Chile. Tramitação do Projeto de Lei Mejor Fonasa. [acessado 2020 jun 30]. Disponível em: https:// www.camara.cl/pley/pley_detalle.aspx?prmI$\mathrm{D}=13719$ \&prmBoletin $=13178-11$.

38. Birn AE, Nervi L. What matters in health (care) universes: delusions, dilutions, and ways towards universal health justice. Globaliz Health 2019; 15(Supl. 1).

Artigo apresentado em 20/11/2020

Aprovado em 03/05/2021

Versão final apresentada em 05/05/2021

Editores-chefes: Romeu Gomes, Antônio Augusto Moura da Silva 